\title{
An interventionist strategy for warehouse order picking: evidence from two case studies
}

\begin{abstract}
As the role of the customer becomes more important in modern logistics, warehouses are required to improve their response to customer orders. To meet the responsiveness expected by customers, warehouses need to shorten completion times. In this paper, we introduce an interventionist order picking strategy that aims to improve the responsiveness of order picking systems. Unlike existing dynamic strategies, the proposed strategy allows a picker to be intervened during a pick cycle to consider new orders and operational disruptions. An interventionist strategy is compared against an existing dynamic picking strategy via a case study. We report benefits both in terms of order completion time and travel distance. This paper also introduces a set of system requirements for deploying an interventionist strategy based on a second case study.
\end{abstract}

Keywords: warehousing, order picking, dynamic strategy

\section{Introduction}

With the emergence of business to consumer (B2C) e-commerce, the role of the customer in modern logistics has dramatically changed (Ramanathan et al., 2014; Manzini et al., 2015b; Yu et al., 2016; McFarlane et al., 2016). Customers increasingly desire to place orders at any hour and to have them delivered at a time convenient to them (De Koster et al., 2007; Lam et al., 2015). As a result, the time available for processing and delivering an order is shortening (Gong and De Koster, 2008; Zhang et al., 2016). In addition to placing orders, customers may legally cancel their orders after placing it (Gong and De Koster, 2008). Moreover, a growing number of companies are offering the option to change the delivery time or location (Amazon, 2015b; Tesco, 2015). Hence warehousing systems must be capable of dealing with an increasing number of disturbances. In a business to business (B2B) environment, changes in production philosophies are now leading manufacturers to use smaller batch sizes, produce more customised products, and attempt to reduce cycle times as much as possible (Davarzani and Norrman, 2015; Lam et al., 2015; De Koster et al., 2007). This has led to a larger product variety and contributed to warehouses' need for shorter response times ( $\mathrm{Li}$ et al., 2017; Gu et al., 2007; Chew and Tang, 1999; Otto and Chung, 2000).

It is, therefore, apparent that improving the response time to customer orders, be it in a B2B or a B2C context, is critical (Van Nieuwenhuyse and De Koster, 2009; Li et al., 2017). Furthermore, the unpredictable and changing nature of customer orders drives the need for modern warehousing systems' responsiveness. To achieve this, warehouses must improve order completion times, should they wish to meet the responsiveness 
expected by customers (Dekker et al., 2004), especially now that same-day delivery is one of the targets for businesses (Amazon, 2015a; Argos, 2015).

Among warehouse activities, order picking is the most labour-intensive and time-consuming one, especially within manual warehouses (Dekker et al., 2004; Bartholdi and Hackman, 2010; Manzini et al., 2015b). As a consequence, reducing the time needed for order picking can be critical for improving responsiveness in a warehouse. Making order picking less laborious is also important to improving efficiency (Ho et al., 2008; Thomas and Meller, 2015). A number of articles have reported that it accounts for as much as 55\% (De Koster et al., 2007; Tompkins et al., 2010), 50-65\% (Ma and Zhao, 2014), 60-65\% (Henn, 2012), or even 60-70\% (Chen et al., 2015) of warehousing costs.

Motivated by this, in this paper we introduce a so-called interventionist order picking strategy that aims to improve the responsiveness of warehouses by minimising the average order completion time (completion time - arrival time). The key idea behind such an "interventionist" strategy is that an order picking system could potentially perform better if it could be intervened by new orders arriving during the order picking process. We use the term 'order picking strategy' to refer to a set of key decisions associated with order picking, i.e. batching, pick-list realising and routing (De Koster et al., 2007). The interventionist order picking strategy is compared against a static strategy as well as against an existing dynamic picking strategy. The strategy is also tested in a trial that aims to identify the key requirements of a system deploying it in practice.

We focus on manual picker-to-part order picking systems as they are the most common systems worldwide (Gong and De Koster, 2008; De Koster et al., 2007; Battini et al., 2016) and are likely to remain so for some time. Manual systems have been reported to reach adoption levels of $80 \%$ in the industry (De Koster et al., 2007; Napolitano, 2012). Although automating the order picking operation is feasible with today's technology, firms often choose manual solutions due to their lower cost and greater flexibility (Grosse et al., 2015; Elbert et al., 2016), leaving aside the risks associated with manual operations (Grosse et al., 2016). Indeed a very recent industrial survey indicated that only $3 \%$ of the respondents use automated picking systems while $60 \%$ still perform traditional paper-based manual picking (Michel, 2016).

The remainder of this paper is structured as follows. In Section 2 we review existing strategies to dynamic order picking. In Section 3, we introduce an interventionist order picking strategy which we then examine in two case studies in Section 4. We conclude with a discussion of the results and future research.

\section{Background}

In this section we review warehouse order picking operations in general as well as the dynamic strategies proposed for managing them. We conclude by presenting a set of limitations of existing dynamic picking strategies. 


\subsection{Order picking}

Order picking is the process of retrieving a stock-keeping unit from its storage location in a warehouse (Petersen and Schmenner, 1999; Roodbergen and De Koster, 2001; Grosse and Glock, 2015). The orderpicking system that controls the order picking operation, can be categorised into two types (Koo, 2008):

- Picker-to-parts system, where the picker travels (walks or drives) along the aisles to the allocated storage locations to retrieve the requested items. Such systems can be either manual or automated depending on whether the system employs humans or machines respectively as the pickers (De Koster et al., 2007).

- Parts-to-picker system, where the requested items are retrieved in an unit load (e.g. pallet or bin) and brought to a pick position (e.g. a depot) for a picker to take the required number of pieces, and after which the remaining load is stored again. Such systems are automated as they employ automated storage and retrieval machines (Manzini et al., 2015b).

In order to maximise the service level provided by order picking systems, researchers have mainly taken two approaches (De Koster et al., 2007; Gu et al., 2010):

1. Storage optimisation (for picking): optimise the way items are stored so that can be easily and quickly retrieved during picking. This is often done via assigning different zones in a warehouse or deciding on specific storage locations of incoming goods (De Koster et al., 2007). Recent research in this area has focused on storing systems capable of handling dynamic and fluctuating demand (Manzini et al., 2015a; Tsamis et al., 2015; Li et al., 2016; Diaz, 2016).

2. Picking optimisation: optimise the order picking operation itself by batching orders together in pick lists, by routing pickers in a warehouse, by deciding when and how sorting will take place and by choosing the appropriate picking system (Davarzani and Norrman, 2015). Recent work in this area has focused in dynamic approaches for order picking (see below), the usage of metaheuristics for complex batching problems (Henn and Wscher, 2012; Pan et al., 2015; Mutingi and Mbohwa, 2017), the impact of the human factor (Grosse and Glock, 2015; Vries et al., 2016), the joint optimisation of batching and routing (Li et al., 2017), and the performance of different automation technologies (Hong et al., 2016; Bortolini et al., 2016; Lamballais et al., 2017).

In this study we focus on the second approach. Depending on the availability of order information, existing studies on order picking generally focus on either a deterministic or a stochastic setting (Van Nieuwenhuyse and De Koster, 2009; Gong and de Koster, 2011; Henn, 2012; Xu et al., 2014). In a deterministic setting, warehouse operations are assumed to use information that is known at the beginning of the planning period (Henn, 2012). Several static models and algorithms have been developed for problems in a deterministic setting (e.g. (Ratliff and Rosenthal, 1983; Elsayed et al., 1993; Van den Berg et al., 1998)), which assume 
perfect information availability about the objective function, and use this information to determine the search direction (Gong and de Koster, 2011).

In a stochastic setting, warehouses receive highly variable information throughout their operations. For example, with respect to customer orders, no information is known in advance about the actual content, size, or the arrival time of an order (Gong and De Koster, 2008). Stochastic settings are more realistic in practice (Van Nieuwenhuyse and De Koster, 2009). Even in a stochastic setting, deterministic models can still provide a good approximation in stable business settings (Gong and de Koster, 2011). However, static, deterministic models may become insufficient or even lead to wrong conclusions in highly uncertain environments such as in systems with strongly fluctuating order patterns and responsive operations (Gong and de Koster, 2011). Hence dynamic strategies are proposed and developed which provide a means of coping with systems that are stochastic, unstable, or inappropriate for deterministic methods. We will review these strategies next.

\subsection{Dynamic strategies in order picking}

A number of dynamic strategies have been suggested for managing warehouse orders in stochastic settings. These strategies generally aim to minimise the time any one order stays in a picking system, referred to as order completion time, turnover time, throughput time or response time (Henn et al., 2012). An overview of this strategies, with example references, is given in Figure 1.

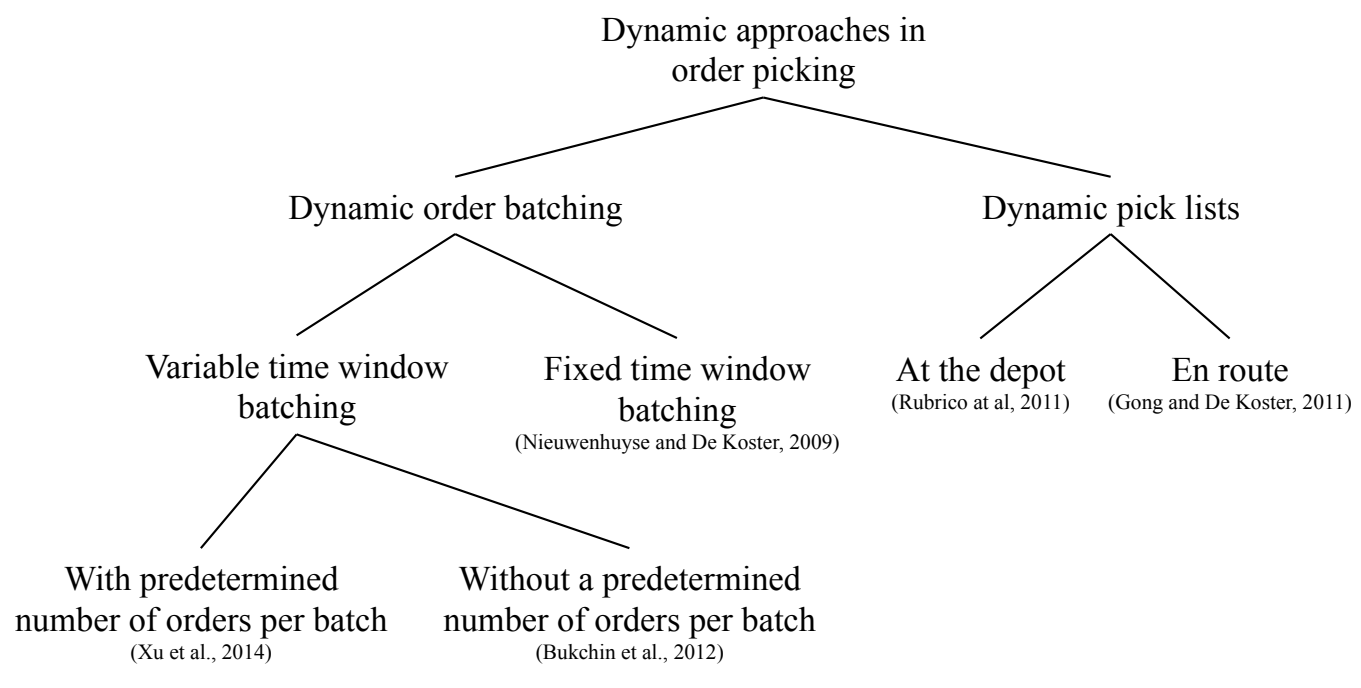

Figure 1: An overview of dynamic strategies in order picking

\subsubsection{Dynamic Order Batching}

Most studies focus on dynamic variations in order batching as opposed to varying the execution of the picking process itself. Order batching is the problem of determining the number of orders to be picked together in one picking tour (Le-Duc and De Koster, 2007; De Koster et al., 2007). More specifically, order batching concerns the partitioning of orders according to time windows or among workers to minimize travel 
distance (Gu et al., 2007). In static (offline) batching, customer orders are known at the beginning of the (short-term) planning period (shift or day) whereas in dynamic (online) batching customer orders become available dynamically over time (Henn, 2012). Dynamic batching takes into account the stochastic nature of customer orders and determines the batch size or the batch time window such that the picking operation can be optimised in view of a given performance criterion (Yu and Koster, 2009). For this reason, two main dynamic batching strategies have been suggested and investigated for different business settings: i) fixed time window batching (FTWB), where a batch contains all orders that have arrived in a fixed time interval (Van Nieuwenhuyse and De Koster, 2009; Henn et al., 2012; Schleyer and Gue, 2012; Zhang et al., 2017). ii) variable time window batching (VTWB), where a picker waits until a predetermined number of orders has been received and can be grouped in a batch (Van Nieuwenhuyse and De Koster, 2009; Xu et al., 2014), or until some other criterion is met (Bukchin et al., 2012; Pérez-Rodríguez et al., 2015). Strategies that consider both FTWB and VTWB have also been studied recently with some promising results (Zhang et al., 2016).

\subsubsection{Dynamic Pick Lists}

In dynamic batching, once a batch is formed and routed, the resulting pick list is passed to a picker to start a new pick cycle. An alternative approach where formed pick lists can change due to the arrival of new orders has also been proposed in the literature (Rubrico et al., 2011). This online rescheduling strategy allows new orders to be incorporated along with orders that have not yet been picked by modifying existing picking schedules. This strategy, along with the aforementioned dynamic batching strategies require all picking locations to be known at the beginning of a pick cycle/route and remain fixed throughout it. As a result, even new customer orders that could be picked by a picker without deviating from his pick route can not be added a pick list once a pick cycle has started. A response to this has been proposed in (Gong and De Koster, 2008), who introduced a system that allows a picker to pick the orders of his current pick list along with those new orders arriving at the picker's current pick position, or arriving further downstream in his route. As a result, the order completion time can be shortened compared with traditional batch picking with optimal batch sizes.

\subsection{Overview of literature}

The conclusions we can draw about current strategies in order picking can be summarised in three key points:

1. In stochastic settings where information is not known in advance, a dynamic approach rather than a static approach is preferred because it can easily adapt to highly uncertain environments.

2. Dynamic order picking can be managed by either batching orders together (before a pick list is assigned to a picker) or by modifying existing pick lists. 
3. Almost all existing dynamic strategies prohibit pick lists from being modified once a pick cycle has started (e.g. (Rubrico et al., 2011; Bukchin et al., 2012)), thus not taking into account orders arriving during a pick cycle.

4. The only dynamic strategy that allows pick lists to be dynamically updated during a pick cycle (Gong and De Koster, 2008), limits updates to be triggered only by orders belonging further downstream on an existing route which traverses the whole warehouse. It also assumes infinite capacity of picking carts.

The strategy we propose in the following section attempts to address each of these points and in particular that of Gong and De Koster (2008). We will do this by developing a strategy which exploits the dynamic order picking algorithm of Lu et al. (2016) at its base (although other routing algorithms could also be considered). The Lu et al. (2016) algorithm extends the routing optimisation algorithm in (Ratliff and Rosenthal, 1983) in a way that allows optimal routing to start from any location in the warehouse. The algorithm takes as input a) a revised set of storage locations to be visited (listed on an updated pick-list), and $b$ ) an arbitrary current location of the picker, and calculates the route the picker should follow in order to minimise the distance travelled. This paper defines, evaluates and tests an overall order picking strategy incorporating the routing algorithm of $\mathrm{Lu}$ et al. (2016). Further this paper will develop policies that implement an interventionist order picking strategy, i.e. policies resulting from making specific decisions with regard to batching, routing and interventions management.

\section{An interventionist order picking strategy}

In this section we introduce an interventionist order picking strategy. We begin by providing a working definition for an an interventionist order picking strategy and we then describe the strategy in more detail. Specific ways the strategy can be implemented are analysed by introducing three order picking policies. We conclude this section with a discussion on the potential applications of the strategy in order picking systems.

\subsection{Definition}

We saw in the previous section that existing dynamic strategies to order picking significantly limit (or do not allow at all) the update of a pick list during a pick cycle (i.e. mid-route). However, in an environment of dynamic picking, a strategy allowing new orders to be added to existing pick lists mid-route and optimally re-routing a picker to pick the remaining orders in his pick list regardless of his current location and route could be expected to improve system performance. This is because some new orders lines could require only a small deviation from a picker's current route thus reducing the average order completion time for a reasonably small increase in travel distance. This has also been suggested elsewhere in the literature (Gong and De Koster, 2008). We therefore introduce the following working definition of an interventionist order picking strategy: 


\section{Definition 1. Interventionist order picking strategy}

A procedure for dynamic order picking that allows a pick cycle to be intervened to update a picker's current pick list regardless of the picker's current location, or route or the storage locations of the items in his pick list.

An interventionist order picking strategy for a single-picker system is illustrated in Figure 2 in comparison to static and to other dynamic batching picking strategies reported in the literature. In this strategy, every time a new order is received in the system, it can potentially interrupt the ongoing pick cycle of a picker so that the new order is added to his existing pick list (subject to capacity constraints of the picking cart). If an intervention takes place, the picker then needs to be re-routed accordingly. Finally, we note that batching decisions before a pick cycle are also important for an interventionist order picking strategy. We discuss these three elements of the strategy (batching, interventions, routing) in more detail in Section 3.2.

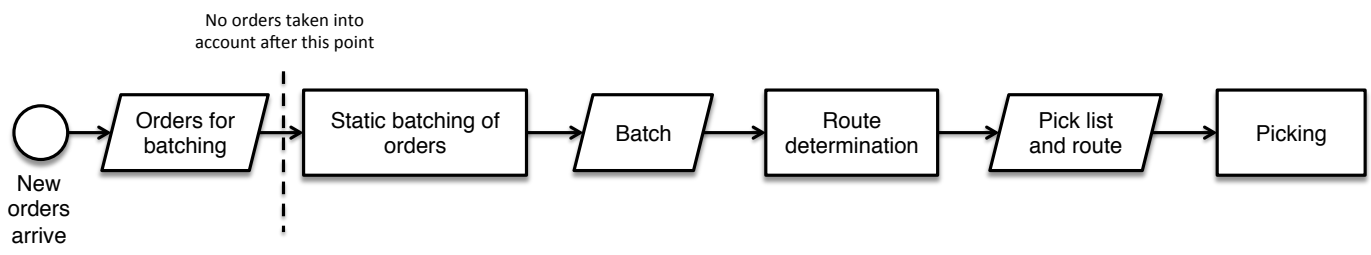

(a) Conventional order picking strategy with static batching.

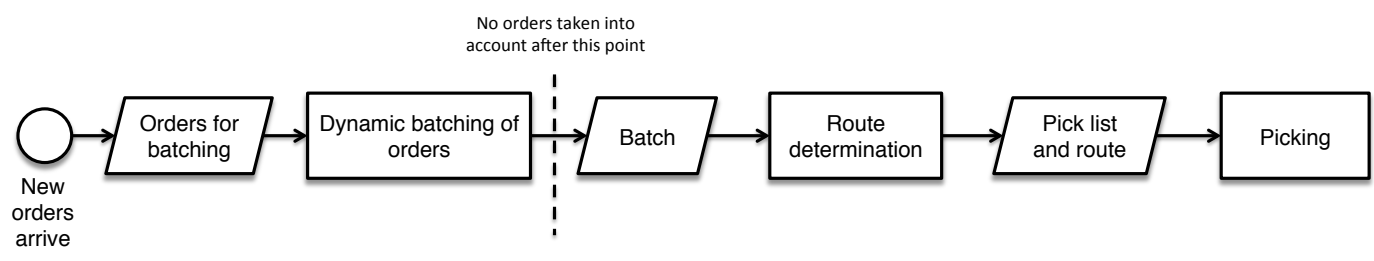

(b) Conventional order picking strategy with dynamic batching.

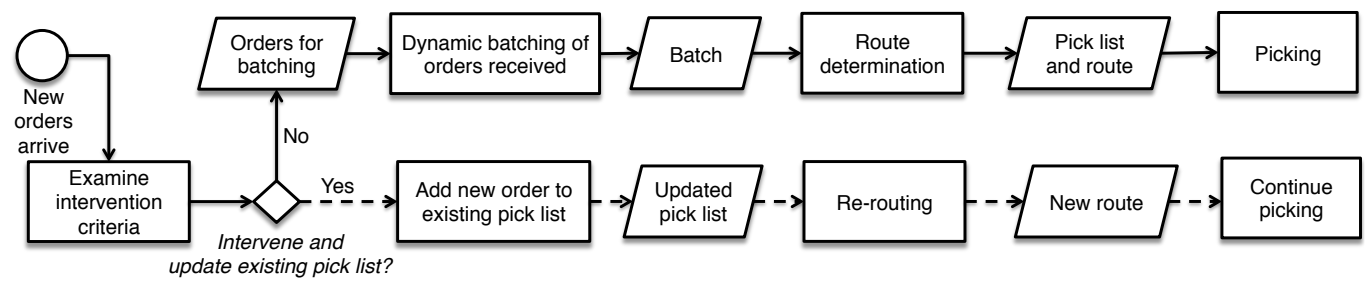

(c) Interventionist order picking strategy with dynamic batching.

Figure 2: A comparison between conventional and interventionist order picking strategies.

\subsection{Regulating an interventionist order picking strategy}

Referring to Figure 2(c), a number of decisions can affect the way an interventionist order picking strategy will operate and perform. Three important decisions are discussed below:

1. What batching policy is best suited to an interventionist order picking strategy? 
Due to the fact that batches (and the associated pick lists) can be updated during the pick cycle, batching should take into account the possibility of new orders arriving during the pick cycle. For example, a picker could leave the depot as soon as a new order enters the system and then update his pick list with new orders during the pick cycle, instead of waiting at the depot for a specific number of orders to arrive. Moreover, a batching policy should attempt to optimise not only the pick list that is currently being picked but also consider future ones. The decision of the picker while at the depot regarding when to start a new pick cycle and which orders to include in a batch can therefore be critical to the performance of an interventionist order picking strategy.

\section{When should a picker's pick list be updated?}

The arrival of a new order or the occurrence of an operational disruption can create an opportunity for updating a pick list in an interventionist order picking strategy. Nevertheless, updating a pick list is not always expected to improve the efficiency of the picking operation. One can imagine a case where the picker is approaching the depot after completing his current pick list when a new order requiring items at the far end of the warehouse arrives. In this case, adding the extra order in the picker's list will significantly increase the average travel distance and turnover time. Hence, a picker could decide to end a pick cycle and return to the depot regardless of the arrival of new orders if this is expected to improve the overall efficiency of the picking system. Therefore, whether a pick list should be updated - in order to include new orders or cancel current ones - can have a big impact on the benefits provided by an interventionist order picking strategy.

\section{How should the new revised route of a picker be (re)calculated?}

Once the pick list is updated, the picker has to be re-routed in the warehouse in a way that will minimise the distance travelled. The calculation of the new route requires an algorithm that allows a picker to start his revised route from an arbitrary current location in the warehouse and end it at the depot after picking all items in his pick list. This type of problem has previously been examined (for example in (Lu et al., 2016)) where an optimal interventionist routing algorithm for a single-block warehouse was introduced and compared against a modified largest-gap heuristic with interventionist pick list. Depending on the layout of the warehouse, other optimal algorithms or heuristics need to be developed to re-route a picker after his pick list is updated.

The fact that under an interventionist order picking strategy a pick list may be updated during a pick cycle, opens the opportunity to investigate several further decisions that are not generally considered under a non-interventionist strategy. For example, it could be investigated whether a picker should move at a variable speed throughout a warehouse. Assuming a random storage allocation and uniform demand for all SKUs, it could be beneficial for pickers to advance more slowly in areas with a high-density of storage locations because it is more likely for the picker to be close to the storage location of a new order arrival. What is more, if one considers the case when there are no orders to pick in the system, under current strategies, the 
picker would be waiting at the depot for an order to arrive. In the interventionist case, it could be considered to have a policy whereby the picker would wait at (or make his way towards) a location in the warehouse that has a higher probability of being the location of items in future orders. Finally, in a multiple-picker setting, the main question concerns the assignment of a new order to an one or more of the pickers.

The graphical model in Figure 3 describes, in simple terms, the process followed every time a new order enters the system under an interventionist order picking strategy (this can be seen as an expansion of Figure 2(c)). For simplicity, we assume a single-picker system.

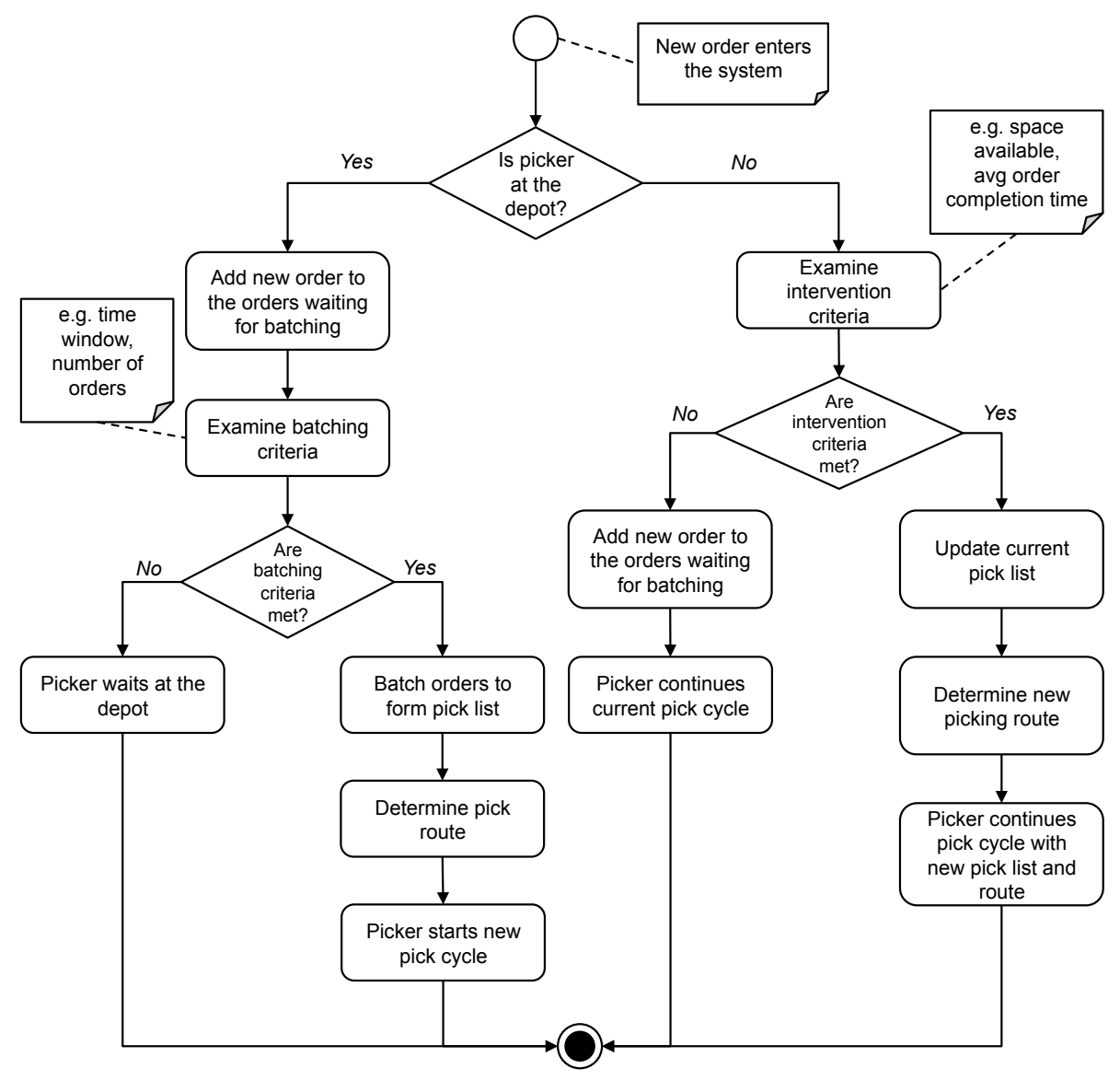

Figure 3: Process followed in an interventionist order picking strategy

\subsection{Picking policy design}

The specific decisions made to regulate an interventionist strategy (i.e. batching, interventions management and routing) can result in a number of different picking policies . In this context, by picking policy, we mean a specified process for controlling the picking activities when new orders enter the system. In interventionist order picking, these policies can vary depending on the scope of interest of the warehouse manager. We identify three such generic cases relating to this scope of interest: 
- Case 1: considering only the impact on the newly arrived order. Adding a new order to the current pick cycle will shorten its completion time as it will not wait for the current pick cycle to finish. This essentially means that the new order will intervene a pick cycle as long as adequate capacity on the picking device is available.

- Case 2: considering the impact on the current pick cycle. Here, the impact of an intervention is measured with regard to the impact it has on all the orders on the pick list of the current pick cycle.

- Case 3: considering the impact on a future pick cycle. This means that the impact of intervention is measured with regard to the impact it has on the orders batched together in a pick list of a future pick cycle.

Based on these cases we now introduce three different policies for interventionist order picking:

1. Interventionist - accept all (IAA): the picker waits for $N$ orders to arrive before starting a new pick cycle. He then accepts all new orders that arrive during a pick cycle as long as there is capacity in his picking cart. This policy is the simplest to implement as the only criterion for intervention is capacity availability. However, it is expected that this policy will not perform well because regular interventions could significantly affect the completion time of older orders that have already been picked.

2. Interventionist - order completion time (IOCT): the picker waits for $N$ orders to arrive before starting a new pick cycle. He then accepts a new order as long as it would not increase the average order completion time above a chosen limit of $X$ seconds. This policy is expected to significantly improve performance as it will allow only those interventions that will decrease average order completion time. The specific value chosen for $X$ is crucial to the policy's success and it can vary considerably between different order arrival rates.

3. Interventionist - re-batching (IRB): the picker waits for $N$ orders to arrive before starting a new pick cycle. When a new order arrives during a pick cycle, the unpicked orders in the system, including those in the current pick list and those pending at the depot, are re-batched such that all unpicked orders in the system form pick lists with orders that are stored in similar locations. This can be done in multiple ways. One of them is by maximising the distance between the centres of gravity of the current pick list and the pending orders waiting to be picked. A more detailed description of this particular way to implement this policy can be found in Appendix A. This policy is also expected to perform well because it takes into account future batches when deciding on interventions.

As one can easily observe, there is a big number of such policies depending on the specific decisions made with regard to batching, interventions management and routing. In general, it is logical to expect that the selection of an interventionist order picking policy will make a difference in performance when there is an adequate number of opportunities for intervention. Hence, such a policy will be more preferable when there 
are neither too few nor too many orders arriving in the system (i.e. balanced range of order arrival rate). In the former case, not many orders will be arriving during a pick cycle to trigger an intervention. In the latter case, a picker starts a new pick cycle with an almost full picking cart due to saturated backlog.

\subsection{Applications of an interventionist order picking strategy}

We conclude this section with a discussion on the potential uses of interventionist order picking. The option to update a picker's pick list mid-route can be beneficial in two cases: $i$ ) when picking is under way and new orders arrive in the system, and $i$ ) when disruptions to the picking process occur. In the first case, a pick list can be updated in order to improve the efficiency of the system mainly in terms of the order completion time which often measures the performance of a dynamic order picking system (Henn et al., 2012). In the second case, updating a pick list could potentially reduce the negative impact of the disruption on the performance of the order picking process. A list of potential uses of an interventionist order picking strategy is given in Table 1.

Table 1: Possible uses of an interventionist order picking strategy (under different picking conditions)

\begin{tabular}{|c|c|c|}
\hline & Space available in picking cart & No space available in picking cart \\
\hline \multirow[t]{6}{*}{$\begin{array}{l}\text { Arrival of } \\
\text { new orders }\end{array}$} & $\begin{array}{l}\text { Allocate new orders to the picker during a } \\
\text { pick cycle }\end{array}$ & $\begin{array}{l}\text { Swap with picked order if urgent or more } \\
\text { profitable order arrives }\end{array}$ \\
\hline & $\begin{array}{l}\text { Swap scheduled order with a new order if } \\
\text { it will improve efficiency }\end{array}$ & \\
\hline & Release a batch faster and add new orders & \\
\hline & while picking & \\
\hline & $\begin{array}{l}\text { Re-batch all orders not yet picked to im- } \\
\text { prove efficiency }\end{array}$ & \\
\hline & $\begin{array}{l}\text { Allocate scheduled order to a different } \\
\text { picker if it improves efficiency }\end{array}$ & \\
\hline \multirow[t]{4}{*}{$\begin{array}{l}\text { Real-time } \\
\text { disruption }\end{array}$} & $\begin{array}{l}\text { Re-route picker to different storage loca- } \\
\text { tion if item is missing }\end{array}$ & Cancel order during a pick cycle \\
\hline & $\begin{array}{l}\text { Update picker's pick list if the contents of } \\
\text { an order are modified by the customer }\end{array}$ & $\begin{array}{l}\text { Allocate scheduled order to a different } \\
\text { picker if disruption occurs }\end{array}$ \\
\hline & Cancel order during a pick cycle & \\
\hline & $\begin{array}{l}\text { Allocate scheduled order to a different } \\
\text { picker if disruption occurs }\end{array}$ & \\
\hline
\end{tabular}

As it can be seen in Table 1, using an interventionist order picking strategy, a pick list can be updated by either adding or removing orders from it because of newly arrived orders, changes/cancellations of received orders, or opportunities to improve (or reduce loss in) performance. 


\section{Warehouse order picking case studies}

We now turn our attention to the performance of an interventionist order picking strategy and the challenges for its deployment in existing order picking systems. Therefore, the aim of this section is twofold: i) to evaluate the benefits of an interventionist order picking strategy and examine the conditions under which it outperforms existing strategies, and ii) to identify the requirements for an information system capable of implementing an interventionist order picking strategy. It is important to look at both of these issues in order to examine both the practical feasibility and the economic viability of deploying an interventionist order picking strategy in practice.

We use two warehouse case studies to examine these issues. The cases are based on the operations of two separate warehouses (denoted A and B) of a collaborative third party logistics provider in China facing the same problem and under much pressure due to recent e-commerce developments in China.

\subsection{Problem description}

The warehouses under consideration in this study are both order fulfilment centres for a number of ecommerce retailers, mainly selling electronic products. Upon receiving orders from the end-customers, the retailers will firstly review the order details (i.e. the correctness of the shipping address, payment details, gift attachment etc.). Once all necessary information is reviewed, orders are forwarded to the warehouses for fulfilling. Both warehouses use manual picker-to-part systems for picking the customer orders. In order to reduce the time needed to prepare an order for despatch, the company owning both of the warehouses wishes to consider allowing its pickers to start a pick cycle while new orders are still being received. The goal of the company is to reduce the time required to pick an order as the lead time available for order preparation is significantly decreasing.

The company would like to:

1. evaluate the benefits offered by an interventionist order picking strategy Also, to understand under what circumstances the proposed strategy outperforms an existing dynamic strategy.

2. understand the main steps for deploying an interventionist order picking strategy in practice and the challenges associated with it. In this way, the company can identify the system requirements that should be considered when deploying an interventionist order picking strategy in a real picking system.

\subsection{Case 1: Interventionist order picking strategy performance evaluation (Warehouse A)}

The first case involves a rectangular warehouse consisting of 300 unique storage locations. It focuses on a performance evaluation of three different policies for use in interventionist order picking introduced in Section 3.3. 


\subsubsection{Experimental design}

To examine the proposed scenario, a warehouse model was designed based on warehouse operations of the collaborating company. The modelled warehouse consists of ten $15 \mathrm{~m}$ long aisles, each containing 30 storage locations ( 15 on each side). The aisles are connected at their endpoints by two cross-aisles. The distance between two consecutive aisles is $3 \mathrm{~m}$. This effectively creates a single-block warehouse. The depot is located in the middle of the bottom cross aisle. The aisles of the warehouse are narrow enough for the picker to be able to retrieve an order without additional time consuming movement other than the picking time per item. For the purposes of this initial study for the company, we assumed that any given order contains a single order line (i.e. a single SKU per order) which is very common in orders placed by consumers online via e-commerce websites. The order arrival time follows a Poisson distribution. We also required each SKU to have its own unique storage location within the warehouse which has been assigned to it randomly. Each SKU takes up the same volumetric space on the picking cart (that has a capacity of 20 items) and there is a uniform demand for all SKUs.

The picking system of the warehouse is a single-picker system, with one picker travelling with a constant speed of $1 \mathrm{~m} / \mathrm{s}$ and requiring $5 s$ (on average) to pick an order. A setup time of $5 \mathrm{~s}$ is included to reflect the time it takes for a picker to retrieve a cart. The setup time is invariant with respect to the pick list size (Van Nieuwenhuyse and De Koster, 2009). A finishing time of $5 s$ per order is also incorporated to reflect the

time it takes to offload all the picked items. Finally, since the demand the company has to fulfil varies, it is required that different order arrival rates ( $\lambda$, measured in number of orders per second) should be examined.

Four policies are compared in this study. The first one is a well-established policy (Van Nieuwenhuyse and De Koster, 2009) used in conventional order picking with dynamic batching strategies (see Figure 2(b)), previously discussed in Section 2.2.1. This policy can be described as follows:

- Dynamic batching policy (DB): the picker waits for $N$ orders to arrive before starting a new pick cycle ( $1 \leq N \leq$ CartCapacity). This is a variable time window batching policy. We will use this policy as the benchmark for the interventionist ones. Note that when $N=20$, the policy is identical to a static one where the picker does not leave the depot unless there are enough orders to fill his picking cart. The routing of the picker from the depot is done in an optimal way based on the seminal algorithm in (Ratliff and Rosenthal, 1983).

The remaining three policies used in our experiments are different policies of an interventionist order picking strategy (denoted IAA, IOCT, IRB) as already described in Section 3.3. In these interventionist policies we allow several updates on a single tour to occur should new orders arrive in the system and provided the criteria for interventions are met. In each of these policies, a picker can be intervened regardless of his current location in the warehouse and at any single point of time he is aware of only the next storage location he needs to visit. Similarly to the dynamic batching policy, the routing of the picker from the depot is done in an optimal way (Ratliff and Rosenthal, 1983). Optimal re-routing from an arbitrary point within 
the warehouse is achieved using the algorithm proposed in (Lu et al., 2016).

\subsubsection{Approach}

In this section, we outline the experiments conducted in this case study. Experiments were conducted in three stages:

Stage 1. Determine suitable order arrival rates: We noticed in the previous section that an interventionist order picking strategy is expected to behave very similarly to a static one, if the picker's backlog exceeds the capacity of the picking cart when he starts a new pick cycle. This is important because if this is the case, once the picker returns to the depot to deliver a pick list, he is immediately given a new full pick list to collect, with no free cart space to intervene with new orders during his pick cycle. The picking system studied here behaves as a single-server queueing model with variable service time depending on the number of orders in a pick list. For $\lambda=0.06$, the service time exceeds the arrival time for 20 orders (360 seconds vs 333 seconds). Hence, there are always going to be at least 20 orders to be picked in the queue (Slack et al., 2013). As a result, the upper limit for $\lambda$ in order for the backlog not to exceed the capacity of the picking cart was set at $\lambda=0.05$ An experimental analysis of the backlog also confirmed this upper limit.

Stage 2. Determine values of parameters for each policy: As the picking polices compared in these experiments are determined by certain parameters, it is necessary to identify the values of those parameters that can offer the best performance for each policy (in terms of AOCT - average order completion time per order). Hence, for each order arrival rate $\lambda=0.01-0.05$, experiments were conducted to determine:

1. For the DB, IAA and IRB policies: the best value for $N$ (number of orders to wait for before starting a new pick cycle). Each policy was tested for the following steps of $N, N=1,5,10,15,20$.

2. For the IOCT policy: the best values $N$ and $X$ (the limit to increased completion time in seconds). This was done by first identifying the best value for $N$ using a large range of $X$ ( $X=150,300,450,600,750)$ and then using that value for $N$ to identify the best value for $X$.

Stage 3. Compare policies: The four policies were compared for each order arrival rate $\lambda=0.01-0.05$, using the values for $N$ and $X$ that yield the minimum average order completion time (AOCT) for each policy. AOCT was used as the key measure as it is a good performance indication of dynamic order picking systems (Henn, 2012; De Koster et al., 2007; Chew and Tang, 1999). Reduced completion times can result in improved service levels and increase the capacity of the warehouse. This set of comparisons emphasises the benefits of the three policies linked to interventionist order picking, as well as determines the best operational conditions (in terms of orders arrival rate) for each policy. 
The experiments ran in a simulation environment developed using NetLogo ${ }^{1}$ and $\mathrm{MATLAB}^{2}$. The features of the logistics company were reflected in the model underpinning this simulation (e.g. layout, operating times, number of storage locations, arrival rates). In our experiments, each of the four policies was tested on the same order portfolio over a duration of seven (simulation) working days to ensure objective comparison. In order to test all possible parameters in each policy we ran more than 1500 unique iterations (each one simulation day long). The number of orders per simulation day ranged from 288 orders for $\lambda=0.01$ to 1440 orders for $\lambda=0.05$.

\subsubsection{Results}

We begin by examining the performance of the four policies in terms of AOCT. In Table 2, the values for the parameters $(N$ and $X)$ that gave the best (smallest) AOCT for each policy at each order arrival rate are given. Notice that for all but one cases, $N=1$ offered the best performance. This means that the picker should start a new pick cycle as long as there is at least one order in the system. In Figure 4, the best performance of each of the three policies based on an interventionist order picking strategy is compared against the best policy based on dynamic batching, in terms of AOCT. A negative value in the plot indicates a reduction in $\mathrm{AOCT}$ relative to $\mathrm{DB}$, i.e. an improvement in performance. The shaded area is the main area of interest.

\begin{tabular}{|c|c|c|c|c|}
\hline$\lambda$ & DB & IAA & IOCT & IRB \\
\hline 0.01 & $N=1$ & $N=1$ & $N=1, \quad X=78$ & $N=1$ \\
\hline 0.02 & $N=1$ & $N=1$ & $N=1, \quad X=90$ & $N=1$ \\
\hline 0.03 & $N=1$ & $N=1$ & $N=1, \quad X=144$ & $N=1$ \\
\hline 0.04 & $N=1$ & $N=1$ & $N=1, \quad X=225$ & $N=1$ \\
\hline 0.05 & $N=1$ & $N=1$ & $N=1, \quad X=380$ & $N=20$ \\
\hline
\end{tabular}

The results in Table 2 and Figure 4 confirm behaviour predicted in Section 3.3:

1. A regulated policy based on an interventionist order picking strategy (i.e. policies IOCT and IRB here) can offer significant benefits over a policy based on a dynamic batching strategy (exceeding $10 \%$ in AOCT reduction) in certain cases. The way interventions are regulated is important for a policy's success as it can seen from the fact that $X$ varies considerably with $\lambda$ for the IOCT policy.

2. An unregulated policy (here IAA) that does not take into account existing orders could have negative results and decrease the overall performance of a picking system.

\footnotetext{
${ }^{1}$ https://ccl.northwestern.edu/netlogo/

${ }^{2}$ http://www.mathworks.co.uk/products/matlab/
} 


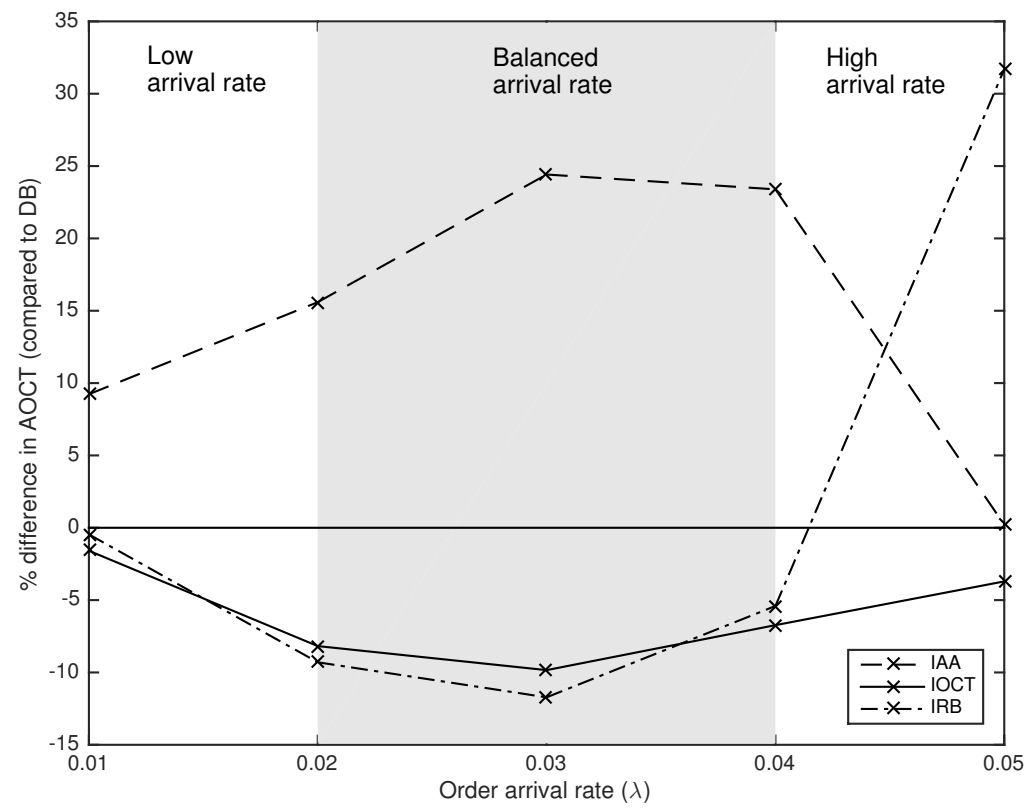

Figure 4: Performance of interventionist order picking strategy based policies against best dynamic batching policy in AOCT

3. The benefits of an interventionist order picking policy are greater when there are adequate opportunities for interventions (here $\lambda=0.02-0.04$ ).

We also notice that when $\lambda=0.05$, IRB performs very poorly compared to the other policies even when $N=20$ and no interventions are allowed. This happens because of the logic of the way the centroid distance is maximised in IRB (see Appendix A). As there are a greater number of orders arriving, the algorithm favours solutions that batch orders at the left and right hand extremities of the warehouse. The AOCT of those in the centre is therefore significantly higher, which reduces the overall performance. At higher values of $\lambda$, it would be better to use an alternative formulation of the algorithm to avoid this effect.

Having demonstrated the benefits of policies using an interventionist order picking strategy in terms of completion time, the same comparison is conducted in terms of the average travel distance (ATD) per order; another key performance indicator which is often considered as a primary objective in warehouse design and optimisation (De Koster et al., 2007; Davarzani and Norrman, 2015). The performance comparison between the same policies previously compared is illustrated in Figure 5. We can make the following observations from Figure 5:

1. Improving the performance of an order picking system in terms of completion time does not necessarily come at a cost in terms of travel distance: compared to DB both IOCT and IRB reduce ATD in all but one cases.

2. The IAA policy's deficiency in terms of AOCT is translated into significant benefits in ATD. Indeed, 
adding more orders to a batch always results in reduced overall travel for any given picking strategy.

3. The significant reduction in ATD for IRB when $\lambda=0.05$ is due to the fact that, according to Table 2, the picker starts a new pick cycle with enough orders to fill his picking cart and he is not intervened with new orders.

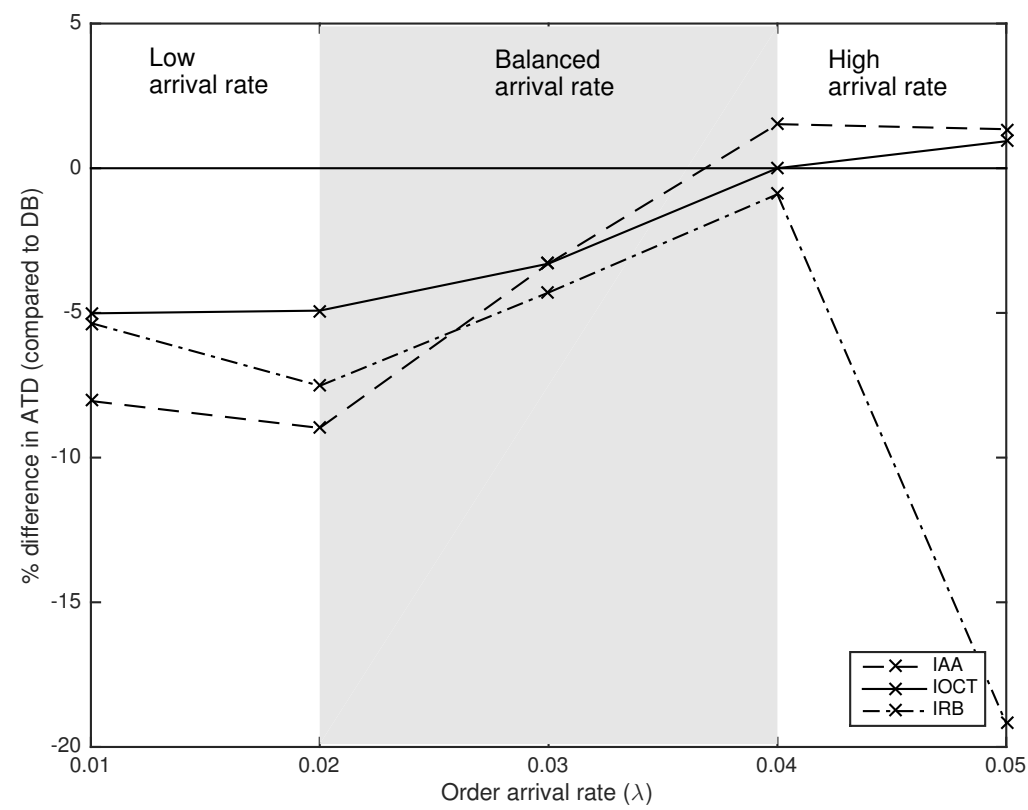

Figure 5: Performance of interventionist order picking strategy based policies against best dynamic batching policy in ATD

In the third part of our analysis, we now focus on another important key performance indicator commonly used in practice: the number of total units picked (Richards and Grinsted, 2013; Staudt et al., 2015). As it can be seen from Figure 6, the best policies in terms of order completion time perform very similarly $( \pm 0.4 \%)$ when it comes to the total units picked in a typical eight-hour working day, indicating that none of them is expected to offer significantly better results than the other. This is to be expected as in our experiments orders do not arrive fast enough in order to create a backlog which in turn could have an impact in the total units picked in a long time period (e.g. 8 hours).

Lastly, we want to compare the interventionist order picking strategy based policies against a policy based on static batching where all orders are known in advance before a new pick cycle begins (as noted before such a strategy is tested here when $N=20$ in the DB policy). This is important as static policies are expected to perform better in terms of ATD as routing can be optimised for all orders in advance. Table 3 compares a static policy with an IOP policy that has performed well for all $\lambda$. The results indicate that the best performing policy is often different for different KPIs. An policy based on interventionist order picking can significantly reduce the order completion time in a warehouse although it requires pickers to travel more. 


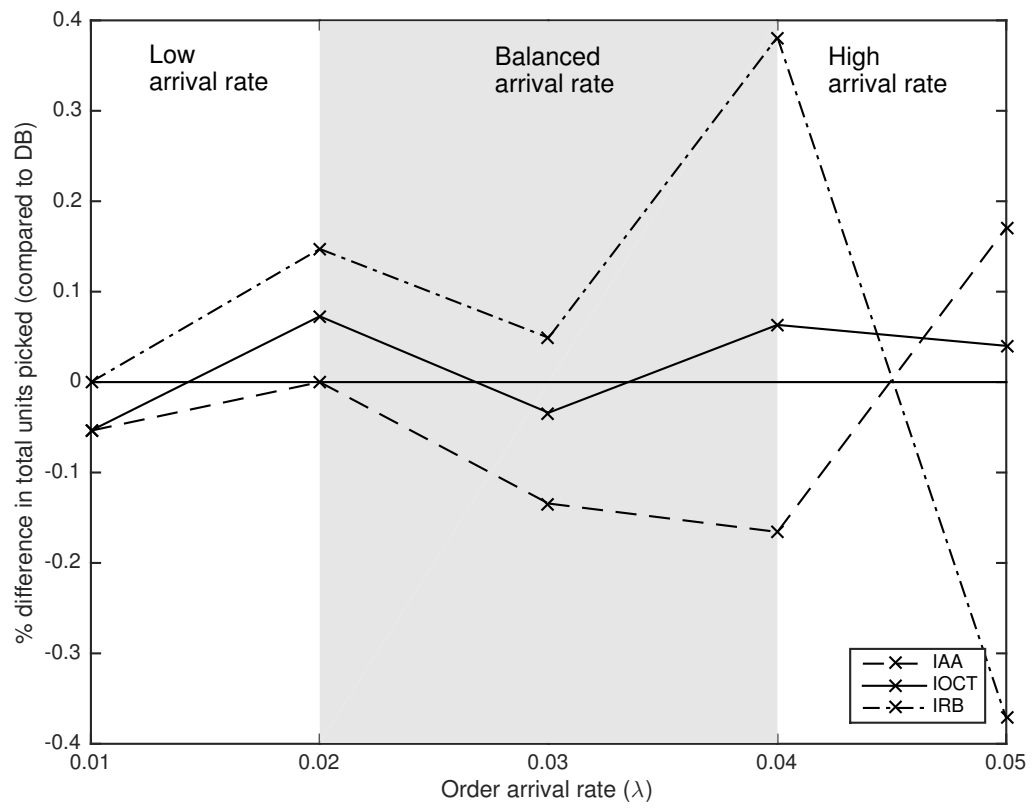

Figure 6: Performance of interventionist order picking strategy based policies against best dynamic batching policy in average number of units picked in a working day

At the same time it can provide some noticeable benefits in terms of overall throughput (i.e. units picked).

Table 3: Comparison between static and interventionist order picking

\begin{tabular}{l|ccc|ccc|ccc|}
\hline & \multicolumn{3}{|c|}{ AOCT } & \multicolumn{3}{c|}{ ATD } & \multicolumn{3}{c|}{ Total Units picked (8h) } \\
\hline$\lambda$ & DB $^{*}$ & IOCT & (if. & DB & IOCT & dif. & DB & IOCT & dif. \\
\hline 0.01 & 1325.57 & 62.59 & $-95 \%$ & 7.93 & 28.22 & $256 \%$ & 268.57 & 279.14 & $3.94 \%$ \\
0.02 & 836.94 & 95.22 & $-89 \%$ & 7.93 & 24.15 & $205 \%$ & 562.86 & 577.71 & $2.64 \%$ \\
0.03 & 677.08 & 152.58 & $-77 \%$ & 7.84 & 19.36 & $147 \%$ & 842.86 & 856 & $1.56 \%$ \\
0.04 & 608.16 & 249.58 & $-59 \%$ & 7.82 & 14.44 & $85 \%$ & 1108.57 & 1123.14 & $1.31 \%$ \\
0.05 & 605.38 & 442.65 & $-27 \%$ & 7.79 & 9.73 & $25 \%$ & 1417.14 & 1423 & $0.41 \%$ \\
\hline
\end{tabular}

* DB policy with $N=20$

** IOCT policy with $N$ and $X$ minimising AOCT (see Table 2)

\subsection{Case 2: Interventionist order picking strategy deployment evaluation (Warehouse B)}

A three-day trial took place in Warehouse B in March 2016, aiming at deploying an interventionist order picking strategy in the existing warehouse picking system. 


\subsubsection{Experimental design}

As a first step in this case study, we needed to capture the current picking process: Upon receiving the orders forwarded from the e-commerce retailers, a warehouse management system (WMS) operator inputs them into the WMS in order to pair the order with the associated storage location. In the WMS, the orders are batched based on the clients, i.e. the e-commerce retailers. As a rule of thumb, the WMS operator normally waits for at least 10 orders, except for urgent orders, before printing a batch into a paper pick list. This is an example of dynamic batching with a fixed minimum number of orders. Once the pick list is printed, the system operator hands it over to an available picker on-site. This picker then picks all the listed products from their storage locations, and transfers them to a packing station. The operation uses a sort-while-pick type of picking, also known as cluster picking (Richards, 2014) using a picking cart that can carry 20 orders. Compared to a sort-after-pick system, a sort-while-pick system allows the company to avoid a separate downstream sorting system after picking, even though it can increase the item extraction time (Gu et al., 2007). The current process of the picking operation is illustrated in Figure 7.

Once the picking process was determined, the layout of the warehouse picking area was converted into a mathematical graph. This allowed us to incorporate the interventionist routing algorithm (Lu et al., 2016) within the interventionist order picking strategy used during the trial. Although there are multiple blocks in the warehouse, each block contains the products from a single client and hence each block can be treated almost as a separate operation. Currently the orders to be picked together are batched per client i.e. each pick list only contains the orders from one client and thus the picking area for this pick list is limited to a single block. The client chosen for our trial was one that sells mostly small products so that the trial could be easily conducted without using special equipment such as a fork-lifter. The layout of the chosen block consists of 7 aisles. Each aisle, except for the first one, has storage locations on both sides. Each row contains 24 storage locations which are 1 meter wide each. The distance between two subsequent aisles is 6 metres. The depot, which is a packing station, is at the left endpoint of the first aisle.

Two further operational parameters were required for the implementation of the interventionist order picking strategy: the picker travelling speed and the item-retrieving time. These were determined taking a number of samples on the first day of our trial. We determined that on average, a picker travels at a slow speed of $1 \mathrm{~m} / \mathrm{s}$ due to the multi-layer picking cart. The item-retrieving time for the warehouse opearations being examined is relatively short $(3 s)$ since each storage location only contains one type of product. Moreover, of particular importance for the regulation of an interventionist order picking strategy is the order arrival rate. This was estimated for different hours of the day using historical data available from the company. The results showed a big range of order arrival rates varying from low rates in the morning and night $(\lambda=0.01-0.03)$ to very high ones around lunch time $(\lambda=0.09-0.13)$ thus making an interventionist order picking strategy more preferable in the morning and night. 


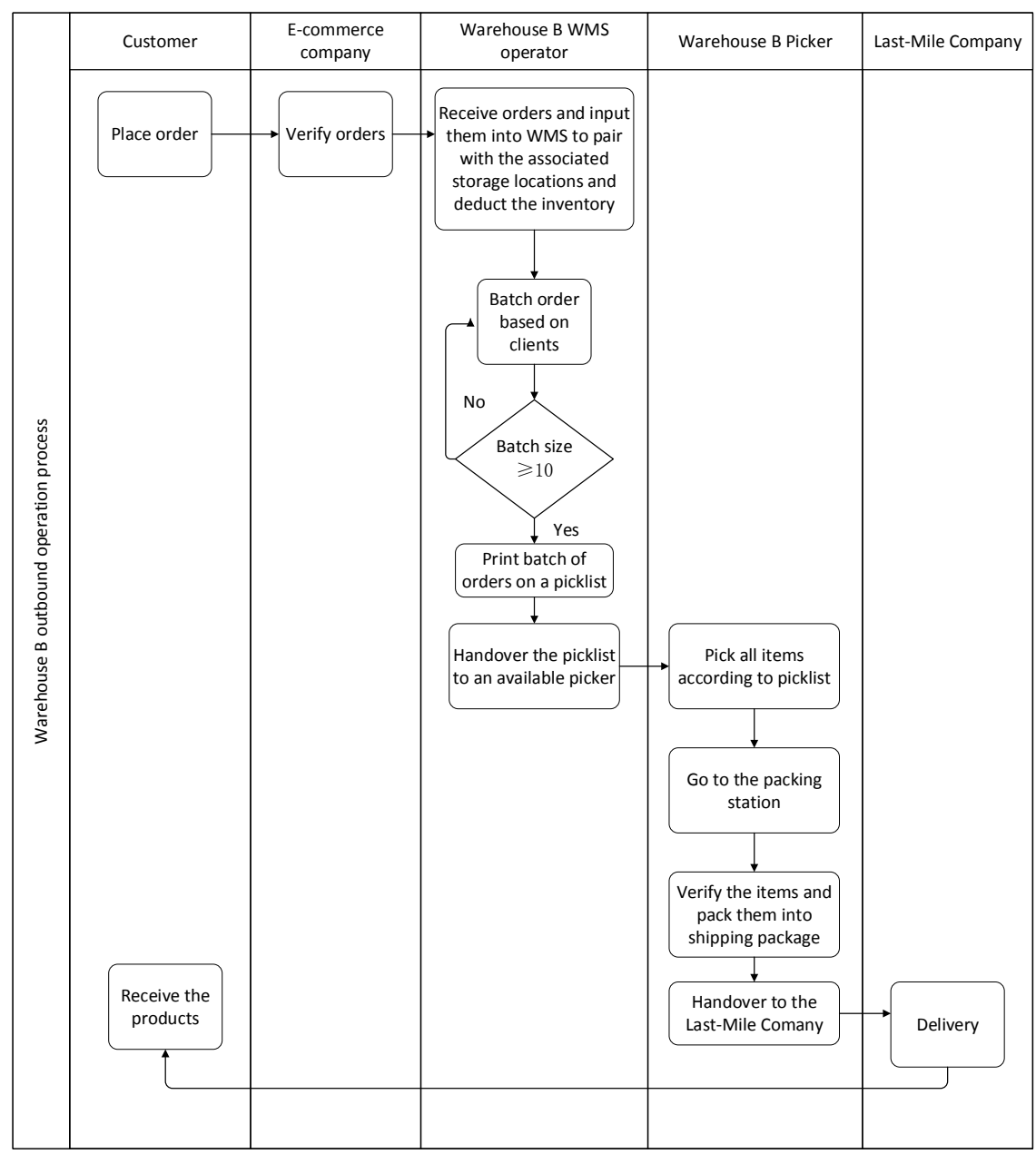

Figure 7: Process diagram of the outbound operation in Warehouse B

\subsubsection{Approach}

In order to conduct the trial, some changes were required to the current picking process. In particular, as interventions are not allowed in the current process, an additional mechanism was added, which enabled the interventions for the interventionist order picking strategy. Specifically, when a new order arrived during a pick cycle, the mechanism enabled the system operator to: i) retrieve the current position of the picker; ii) decide whether the picker should be intervened by the new order; iii) inform the picker about the decision with the pick list and associated picking route. In this stage, some re-programming of our developed interventionist order picking software systems was required to allow the WMS operator to manually enter the order information. The process of the picking operation using an interventionist order picking strategy is illustrated in Figure 8. Comparing Figures 7 and 8 it can be seen that in the interventionist order picking strategy the picker's actions can be dynamically change during a pick cycle with the help of the WMS.

Lastly, we conducted the trial during selected hours with a suitable order arrival rate (i.e. an order arrival 


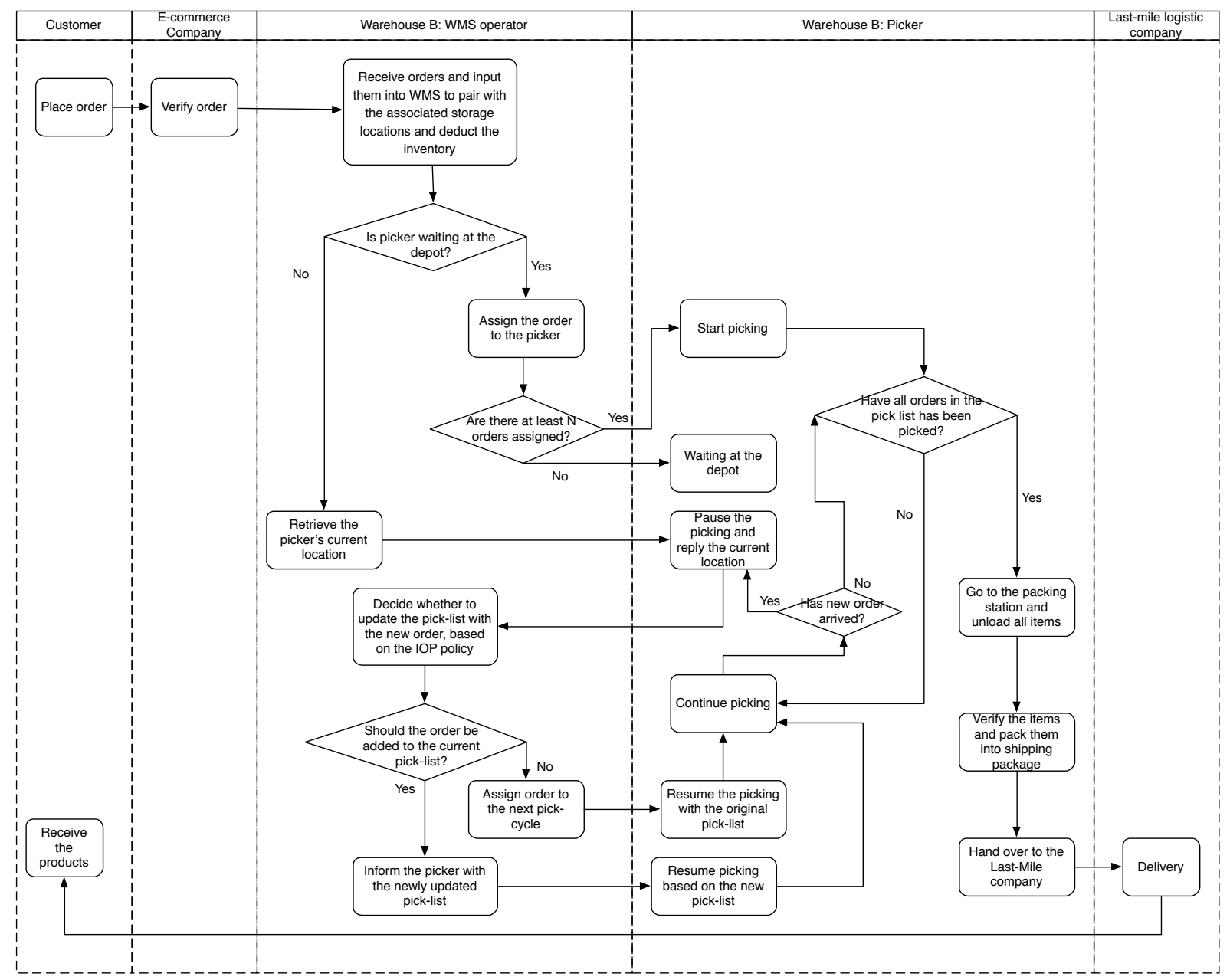

Figure 8: Process diagram of the outbound operation using interventionist order picking strategy

rate that allowed interventions to take place). Due to the fact that the picking operation is paper-based in this case, we equipped the picker with an instant message app on a smartphone, to inform him about the pick list and display the picking route. The picker could also use this instant message app to inform the system operator with his current location and status.

\subsubsection{Results}

As a result of this case study, an interventionist order picking strategy was successfully deployed within an existing picking system. In the new picking system, new orders were dynamically added to existing pick lists during a pick cycle and were communicated to human pickers in real time. During the trial, a number of challenges were identified when attempting to deploy an interventionist order picking strategy within an existing picking system. In this section we introduce five key requirements for successfully implementing an interventionist order picking strategy.

Requirement 1. Order arrivals and disruptions to be continuously tracked 
New orders and/or operational disruptions should be triggered within the system the moment they happen instead of entering the system in waves/large batches as it is common in practice. This is necessary to examine if an intervention is feasible and useful. In our trial, we observed four different time stamps regarding the order's arrival which created confusion in the deployment of an interventionist order picking strategy: i) time an order was placed by the end customer, ii) time payment was received, iii) time an order was reviewed and confirmed by the retailer, and $i v$ ) time an order was forwarded to the warehousing company. On top of that, there might be a time lag between any two time stamps. For instance, we observed a 30-minute lag between the time an order was reviewed and the time it arrived in the warehouse, due to the fact that orders were forwarded in batches (i.e. the first reviewed order will need to wait for the last order in this batch to be reviewed before they can all be forwarded to the warehouse).

Requirement 2. Pickers and picking processes to be tracked

The system should be able to track pickers as they move around the warehouse in order to know their current location as well as the status of the picking process they are executing (i.e. what items have been picked, what items have yet to be picked etc.). This is required in order to evaluate whether to intervene a pick cycle but also in order to inform the picker about his next steps. For the needs of our trial, the picker interacted with the system operator during a pick cycle using an instant message application, in order to report his current location and task. Other solutions for real-time indoors tracking can be used although their capabilities should be carefully examined. A practical work-around is to allow interventions only when the picker is at a storage location doing picking. In this way, the picker can report his current location while picking an item, e.g. by scanning its barcode and/or the barcode of the storage location.

Requirement 3. Pickers to be notified about changes and next steps during a pick process

The system should update the pick lists if and when new orders need to be added and inform pickers about any changes to their pick list and route. This requires appropriate technology to notify a picker in real time during a pick cycle such as tablet or wearable computers and hand-held devices that can display electronic pick lists. Paper-based systems that are common in practice are not suitable for frequent changes even though simple walkie talkies can be used to communicate with a picker. In our trial, we used an instant message app on a smart phone via which the system operator would send the storage location the picker needs to visit to the picker's phone. A challenge here is to find appropriate ways to display notifications and routing advice to a human operator - especially in cases where multiple interventions take place during a pick cycle - as this can prove to be very confusing even for experienced pickers. If interventions are permitted to happen only when the picker completes a pick at a storage location, the system could simply display the next storage location to be visited and the exact route that should be followed to get there.

Requirement 4. Decisions about interventions to be made and executed in limited time

An interventionist order picking strategy often requires the execution of optimisation processes that can be time consuming. For example, the impact of introducing a new order on the delivery of other orders in 
a pick cart needs to be examined before updating a pick list. The same can apply in the calculation of the new route a picker should follow after an intervention. Both tasks take significant computational time. The implementation of an interventionist order picking strategy within a corporate IT environment should take into account the criticality of timely decision making and execution.

Requirement 5. An interventionist order picking strategy needs to be adapted frequently as operating conditions change

The results presented in the first case study demonstrate that updating a picker's pick list mid-route can be beneficial. Nevertheless, we saw that the way an interventionist order picking strategy is regulated (i.e. by deciding how batching, interventions and routing are managed) has a big impact on the performance of the strategy. Depending on the order arrival rate and other operational parameters of the warehouse, the system should be able to estimate a good (if not the best) way to regulate an interventionist order picking strategy. Moreover, due to the fact that the order arrival rate can change frequently, the system should adapt to avoid problems resulting from a poor regulation. In our trial, we observed cases where the order arrival rate could change even during a single hour. An accurate estimation of the order arrival rate and a careful adaptation of the system is therefore central for the successful deployment of an interventionist order picking strategy.

\subsection{Key findings and discussion}

The results of our first case study indicate that an interventionist order picking strategy can significantly improve warehouse performance in terms of average order completion time (Figure 4). The strategy also decreases average travel distance compared to an existing dynamic picking strategy (Figure 5). The strategy is expected to be more beneficial when the frequency of order arrivals supports interventions during a pick cycle. Conversely, the strategy might be less appropriate for warehouses with a large number of incoming orders per picker. More generally, we have also demonstrated the importance of properly regulating the strategy in terms of batching and interventions management.

The order arrival rates identified in Section 4.2 as being most suitable for an interventionist order picking strategy are based on the single case studied. Nevertheless, our analysis has demonstrated that if demand levels permit interventions (e.g. when backlog is not often saturated), this can be used to reduce order completion times. Further work is required to determine how to generalise these results.

It should be noted here that minimising order completion times can often be at odds with minimising travel distance in a warehouse. Indeed, starting a new pick cycle with enough orders to fill a picking cart will almost always result in much shorter distances travelled compared to a cycle with multiple interventions. On the other hand, travelling more to pick new orders during a pick cycle can result in more orders being completed faster as unnecessary waiting can be minimised. As discussed in Section 1, we believe that current business trends have led to order completion time becoming a very important factor for warehouse operations. 
Our analysis in the second case study indicates that warehouse management systems capable of providing high-accuracy tracking information about orders, disruptions and pickers are required for deploying an interventionist order picking strategy. Additionally, real-time communication with a human picker should be available (e.g. hand-held devices, tablets or wearable technologies). For many warehouses, this indicates a significant investment cost for developing or buying such technology (Reaidy et al., 2015). Nevertheless, there are many examples of systems in current practice that collect real-time tracking information and could easily implement an interventionist order picking strategy. Providers of these technologies could be among the first ones testing an interventionist order picking strategy and examining its benefits in their business case.

On the issue of human-system interaction, it is important to consider the difficulties human pickers face when they have to follow a complex route, especially if this route dynamically changes during a pick cycle. As it has been shown previously, optimal routes often seem illogical, complex or unfamiliar to human pickers (Petersen and Aase, 2004; Gademann and Velde, 2005; De Koster et al., 2007; Henn, 2012) and there is empirical knowledge that pickers deviate from them (Elbert et al., 2016). User-friendly interfaces between pickers and warehouse management systems need to be developed in order to minimise the effort needed by a picker, e.g. by showing only one picking location at a time. An alternative approach could be to consider route simplicity as a criterion for determining interventions as familiarity often reduces travel time (Grosse and Glock, 2015).

\section{Conclusions}

In this paper we introduced a new dynamic strategy for order picking that allows route and pick list updating during a pick cycle. In an interventionist order picking strategy, a pick list can be updated by either adding or removing orders from it because of newly arrived orders, changes/cancellations of received orders, or opportunities to improve (or reduce loss in) performance. The work reported here complements previous work in dynamic order picking (Lu et al., 2016) which focused specifically on an algorithm for dynamic routing. This paper provides a more general setting which incorporates such routing algorithms in addition to picker coordination approaches into appropriate order picking strategies.

The performance analysis presented in this paper is based on the operational parameters of the case example of our partner company. The results of our study confirm that a regulated interventionist order picking strategy can decrease the order completion time without at the same time resulting in increased

levels of the distance travelled. Further more detailed studies are required to determine specific parameters for deploying an interventionist order picking strategy in warehouses with different layouts, storage policies, picking carts capacities and demand patterns.

The impact of an improvement in AOCT can be threefold for a warehousing company. Firstly, it can have an impact on customer satisfaction in cases where customers are waiting in a physical store to collect their orders. This is a common example in e-commerce where customers acan place their orders online and 
collect them at their convenience in a physical store. Secondly, it can be used to prioritise urgent orders that have to leave the warehouse with limited time available. Finally, and perhaps most importantly, an interventionist order picking strategy can reduce the overall completion time of the preparation of an order because picking is often the bottleneck and reducing the time it takes to pick an order can reduce the time required to have it ready for despatch.

Three areas are of particular interest for future research in order to evaluate the strategy in an even more realistic context: firstly, the investigation of the strategy's operation and performance in a multiple-picker system. New opportunities and challenges arise in a multiple-picker system such as the allocation of a new order to a picker or the specification of the optimal number of pickers to be used. It also allows for greater demand rates to be examined. Secondly, the analysis of the interventionist strategy's responsiveness under various order lead times. Depending on the business case, the deadline of a warehouse order might vary from a few minutes — when a customer waits at a collection point — to a few hours — when a last-mile delivery truck visits a warehouse once a day. Last but not least, the development of analytical models are required, that would determine the way an interventionist order picking strategy should be regulated. Research in this area includes batching and routing algorithms, picker release and picker allocation models and methods for interventions management.

\section{References}

Amazon, 2015a. Amazon same-day delivery. Available online at http://www.amazon.com/b?node= 8729023011. Accessed November 2015.

Amazon, 2015b. On-line shopping change order information page. Available online at http://www. amazon . co.uk/gp/help/customer/display.html?nodeId=201168510. Accessed November 2015.

Argos, 2015. Argos fast track. Available online at https://www.argos.co.uk/static/StaticDisplay/ includeName/fasttrack.htm. Accessed November 2015.

Bartholdi, J.J., Hackman, S.T., 2010. Warehouse \& distribution science: release 0.92. The Supply Chain and Logistics Institute, School of Industrial and Systems Engineering, Georgia Institute of Technology, Atlanta, GA.

Battini, D., Calzavara, M., Persona, A., Sgarbossa, F., 2016. Additional effort estimation due to ergonomic conditions in order picking systems. International Journal of Production Research In press. DOI:10.1080/00207543.2016.1190879.

Van den Berg, J.P., Sharp, G.P., Gademann, A., Pochet, Y., 1998. Forward-reserve allocation in a warehouse with unit-load replenishments. European Journal of Operational Research 111, 98-113. 
Bortolini, M., Faccio, M., Ferrari, E., Gamberi, M., Pilati, F., 2016. Time and energy optimal unit-load assignment for automatic S/R warehouses. International Journal of Production Economics In press. DOI: 10.1016/j.ijpe.2016.07.024.

Bukchin, Y., Khmelnitsky, E., Yakuel, P., 2012. Optimizing a dynamic order-picking process. European Journal of Operational Research 219, $335-346$.

Chen, T.L., Cheng, C.Y., Chen, Y.Y., Chan, L.K., 2015. An efficient hybrid algorithm for integrated order batching, sequencing and routing problem. International Journal of Production Economics 159, 158-167.

Chew, E.P., Tang, L.C., 1999. Travel time analysis for general item location assignment in a rectangular warehouse. European Journal of Operational Research 112, 582-597.

Davarzani, H., Norrman, A., 2015. Toward a relevant agenda for warehousing research: literature review and practitioners' input. Logistics Research 8, 1.

De Koster, R., Le-Duc, T., Roodbergen, K.J., 2007. Design and control of warehouse order picking: A literature review. European Journal of Operational Research 182, 481-501.

Dekker, R., De Koster, B.M., Roodbergen, K.J., Van Kalleveen, H., 2004. Improving order-picking response time at Ankor's warehouse. Interfaces 34, 303-313.

Diaz, R., 2016. Using dynamic demand information and zoning for the storage of non-uniform density stock keeping units. International Journal of Production Research 54, 2487-2498.

Elbert, R.M., Franzke, T., Glock, C.H., Grosse, E.H., 2016. The effects of human behavior on the efficiency of routing policies in order picking: The case of route deviations. Computers \& Industrial Engineering In press. DOI: 10.1016/j.cie.2016.11.033.

Elsayed, E.A., Lee, M.K., Kim, S., Scherer, E., 1993. Sequencing and batching procedures for minimizing earliness and tardiness penalty of order retrievals. International Journal of Production Research 31, 727738.

Gademann, N., Velde, V.D.S., 2005. Order batching to minimize total travel time in a parallel-aisle warehouse. IIE Transactions 37, 63-75.

Gong, Y., De Koster, R.B., 2008. A polling-based dynamic order picking system for online retailers. IIE Transactions 40, 1070-1082.

Gong, Y., de Koster, R.B.M., 2011. A review on stochastic models and analysis of warehouse operations. Logistics Research 3, 191-205.

Grosse, E.H., Glock, C.H., 2015. The effect of worker learning on manual order picking processes. International Journal of Production Economics 170, Part C, 882 - 890. 
Grosse, E.H., Glock, C.H., Jaber, M.Y., Neumann, W.P., 2015. Incorporating human factors in order picking planning models: framework and research opportunities. International Journal of Production Research $53,695-717$.

Grosse, E.H., Glock, C.H., Neumann, W.P., 2016. Human factors in order picking: a content analysis of the literature. International Journal of Production Research In press. DOI:10.1080/00207543.2016.1186296.

Gu, J., Goetschalckx, M., McGinnis, L.F., 2007. Research on warehouse operation: A comprehensive review. European Journal of Operational Research 177, 1-21.

Gu, J., Goetschalckx, M., McGinnis, L.F., 2010. Research on warehouse design and performance evaluation: A comprehensive review. European Journal of Operational Research 203, 539-549.

Henn, S., 2012. Algorithms for on-line order batching in an order picking warehouse. Computers \& Operations Research 39, 2549-2563.

Henn, S., Koch, S., Wäscher, G., 2012. Order batching in order picking warehouses: A survey of solution approaches, in: Manzini, R. (Ed.), Warehousing in the Global Supply Chain. Springer London, pp. 105137.

Henn, S., Wscher, G., 2012. Tabu search heuristics for the order batching problem in manual order picking systems. European Journal of Operational Research 222, 484-494.

Ho, Y.C., Su, T.S., Shi, Z.B., 2008. Order-batching methods for an order-picking warehouse with two cross aisles. Computers \& Industrial Engineering 55, 321-347.

Hong, S., Johnson, A.L., Peters, B.A., 2016. Order batching in a bucket brigade order picking system considering picker blocking. Flexible Services and Manufacturing Journal 28, 425-441.

Koo, P.H., 2008. The use of bucket brigades in zone order picking systems. OR Spectrum 31, 759.

Lam, H., Choy, K., Ho, G., Cheng, S.W., Lee, C., 2015. A knowledge-based logistics operations planning system for mitigating risk in warehouse order fulfillment. International Journal of Production Economics 170, Part C, 763-779.

Lamballais, T., Roy, D., Koster, M.D., 2017. Estimating performance in a robotic mobile fulfillment system. European Journal of Operational Research 256, 976-990.

Le-Duc, T., De Koster, R.B.M., 2007. Travel time estimation and order batching in a 2-block warehouse. European Journal of Operational Research 176, 372-388.

Li, J., Huang, R., Dai, J.B., 2017. Joint optimisation of order batching and picker routing in the online retailers warehouse in China. International Journal of Production Research 55, 447-461. 
Li, J., Moghaddam, M., Nof, S.Y., 2016. Dynamic storage assignment with product affinity and abc classification - a case study. The International Journal of Advanced Manufacturing Technology 84, 21792194.

Lu, W., McFarlane, D., Giannikas, V., Zhang, Q., 2016. An algorithm for dynamic order-picking in warehouse operations. European Journal of Operational Research 248, 107-122.

Ma, T., Zhao, P., 2014. A review of algorithms for order batching problem in distribution center, in: International Conference on Logistics Engineering, Management and Computer Science, pp. 172-175.

Manzini, R., Accorsi, R., Gamberi, M., Penazzi, S., 2015a. Modeling class-based storage assignment over life cycle picking patterns. International Journal of Production Economics 170, Part C, 790-800. Decision models for the design, optimization and management of warehousing and material handling systems.

Manzini, R., Bozer, Y., Heragu, S., 2015b. Decision models for the design, optimization and management of warehousing and material handling systems. International Journal of Production Economics 170, Part C, 711-716. Editorial.

McFarlane, D., Giannikas, V., Lu, W., 2016. Intelligent logistics: Involving the customer. Computers in Industry $81,105-115$.

Michel, R., 2016. 2016 warehouse/DC operations survey: Ready to confront complexity. Available online at http://www. logisticsmgmt.com/article/2016_warehouse_dc_operations_survey_ready_to_ confront_complexity. Accessed February 2017.

Mutingi, M., Mbohwa, C., 2017. Optimizing Order Batching in Order Picking Systems: Hybrid Grouping Genetic Algorithm. Springer International Publishing, Cham. pp. 121-140.

Napolitano, M., 2012. 2012 warehouse/DC operations survey: Mixed signals. Available online at http:// www.logisticsmgmt.com/article/2012_warehouse_dc_operations_survey_mixed_signals. Accessed February 2017.

Otto, J.R., Chung, Q., 2000. A framework for cyber-enhanced retailing: Integrating e-commerce retailing with brick-and-mortar retailing. Electronic Markets 10, 185-191.

Pan, J.C.H., Shih, P.H., Wu, M.H., 2015. Order batching in a pick-and-pass warehousing system with group genetic algorithm. Omega 57, Part B, 238-248.

Pérez-Rodríguez, R., Hernández-Aguirre, A., Jöns, S., 2015. A continuous estimation of distribution algorithm for the online order-batching problem. The International Journal of Advanced Manufacturing Technology 79, 569-588.

Petersen, C.G., Aase, G., 2004. A comparison of picking, storage, and routing policies in manual order picking. International Journal of Production Economics 92, 11-19. 
Petersen, C.G., Schmenner, R.W., 1999. An evaluation of routing and volume-based storage policies in an order picking operation. Decision Sciences 30, 481-501.

Ramanathan, R., George, J., Ramanathan, U., 2014. The Role of Logistics in E-commerce Transactions: An Exploratory Study of Customer Feedback and Risk. Springer London, London. pp. 221-233.

Ratliff, H.D., Rosenthal, A.S., 1983. Order-picking in a rectangular warehouse: a solvable case of the traveling salesman problem. Operations Research 31, 507-521.

Reaidy, P.J., Gunasekaran, A., Spalanzani, A., 2015. Bottom-up approach based on Internet of Things for order fulfillment in a collaborative warehousing environment. International Journal of Production Economics 159, 29-40.

Richards, G., 2014. Warehouse Management: A complete guide to improving efficiency and minimizing costs in the modern warehouse. Kogan Page Publishers.

Richards, G., Grinsted, S., 2013. The Logistics and Supply Chain Toolkit: Over 90 Tools for Transport, Warehousing and Inventory Management. Kogan Page Publishers.

Robertson, B., 2015. Toward the implementation of interventionist strategies in warehouse picking. Master's thesis. University of Cambridge.

Roodbergen, K.J., De Koster, R., 2001. Routing order pickers in a warehouse with a middle aisle. European Journal of Operational Research 133, 32-43.

Rubrico, J.I., Higashi, T., Tamura, H., Ota, J., 2011. Online rescheduling of multiple picking agents for warehouse management. Robotics and Computer-Integrated Manufacturing 27, 62-71.

Schleyer, M., Gue, K., 2012. Throughput time distribution analysis for a one-block warehouse. Transportation Research Part E: Logistics and Transportation Review 48, 652-666.

Slack, N., Brandon-Jones, A., Johnston, R., 2013. Operations Management. seventh ed., Pearson.

Spears, W.M., Anand, V., 1991. A study of crossover operators in genetic programming, in: Ras, Z.W., Zemankova, M. (Eds.), Methodologies for Intelligent Systems. ISMIS 1991. Lecture Notes in Computer Science (Lecture Notes in Artificial Intelligence). Springer Berlin Heidelberg, Berlin, Heidelberg. volume 542, pp. 409-418.

Staudt, F.H., Alpan, G., Mascolo, M.D., Rodriguez, C.M.T., 2015. Warehouse performance measurement: a literature review. International Journal of Production Research 53, 5524-5544.

Tesco, 2015. On-line shopping help page. Available online at http://www.tesco.com/groceries/help/ default . aspx?name=myOrder\&rel=help. Accessed November 2015. 
Thomas, L.M., Meller, R.D., 2015. Developing design guidelines for a case-picking warehouse. International Journal of Production Economics 170, Part C, 741-762.

Tompkins, J.A., White, J.A., Bozer, Y.A., Tanchoco, J.M.A., 2010. Facilities planning. John Wiley \& Sons.

Tsamis, N., Giannikas, V., McFarlane, D., Lu, W., Strachan, J., 2015. Adaptive storage location assignment for warehouses using intelligent products, in: Borangiu, T., Thomas, A., Trentesaux, D. (Eds.), Service Orientation in Holonic and Multi-agent Manufacturing. Springer International Publishing. volume 594 of Studies in Computational Intelligence, pp. 271-279.

Van Nieuwenhuyse, I., De Koster, R., 2009. Evaluating order throughput time in 2-block warehouses with time window batching. International Journal of Production Economics 121, 654-664.

Vries, J.D., de Koster, R., Stam, D., 2016. Exploring the role of picker personality in predicting picking performance with pick by voice, pick to light and RF-terminal picking. International Journal of Production Research 54, 2260-2274.

Xu, X., Liu, T., Li, K., Dong, W., 2014. Evaluating order throughput time with variable time window batching. International Journal of Production Research 52, 2232-2242.

Yu, M., Koster, R.B.D., 2009. The impact of order batching and picking area zoning on order picking system performance. European Journal of Operational Research 198, 480-490.

Yu, Y., Wang, X., Zhong, R.Y., Huang, G.Q., 2016. E-commerce logistics in supply chain management: Practice perspective. Procedia CIRP 52, 179-185. The Sixth International Conference on Changeable, Agile, Reconfigurable and Virtual Production.

Zhang, J., Wang, X., Chan, F.T., Ruan, J., 2017. On-line order batching and sequencing problem with multiple pickers: A hybrid rule-based algorithm. Applied Mathematical Modelling 45, 271-284.

Zhang, J., Wang, X., Huang, K., 2016. Integrated on-line scheduling of order batching and delivery under B2C e-commerce. Computers \& Industrial Engineering 94, 280-289.

\section{Appendix A. A policy based on interventionist order picking with re-batching}

This policy batches orders together while a picker is mid-route so that the resulting pick lists contain orders which have storage locations that are in close proximity to one another. The justification for this policy is that it is reasonable to suppose that because orders are more closely packed, the travel time per pick list would reduce. Furthermore, this batching policy variant has been designed so that if a new order arrives and it is initially decided not to place it in the current pick list, this will be reconsidered when subsequent new orders arrive mid-tour. Hence, this policy takes into consideration all unpicked orders when a new order arrives in view of forming a better pick list. 
This policy consists of creating a pick list such that when a new order arrives, the picker is sent an updated current pick list which maximises the Euclidean distance between the centroid of the storage locations of the newly updated pick list and the centroid of the storage locations of the orders to be picked in subsequent picking tours. The centroid for the newly updated pick list is calculated from the co-ordinates of $i$ ) the orders that have already been picked on the current picking tour, $i i$ ) the depot location (as the picker must return there), and $i i i$ ) the orders that still need to be picked in the newly updated pick list.

The centroid of the pending pick list items is calculated from the depot location (which is factored in twice because the picker must leave from and return there) and the location of any unpicked orders that have not been added to the newly updated current pick list. The picker in effect is constantly updated with a pick list of items which are grouped as closely as possible to each other, maximising the effective use of his time by minimising the distance between items.

A genetic algorithm was used to determine which unpicked orders should be included in the newly updated pick list and those which should be picked at a later date. The chromosomes are designed such that there is a binary gene for every unpicked order in the system. An unpicked order gene that has a value of 0 will be picked later, while a gene value of 1 denotes an order in the newly updated pick list. For example, in Figure A.9, orders 5, 14, and 16 will be added to the newly updated pick list whereas orders 8 and 16 will not. The genetic algorithm uses a uniform crossover operation (Spears and Anand, 1991), mutation (where a gene of a child chromosome has the chance of changing its binary value), migration (where genes take the value of the preceding genes in the chromosome), elitism (where a fraction of the best performing chromosomes of the previous population are kept for the next generation of chromosomes), and stochastic universal sampling whereby the best performing chromosomes are more likely to be selected for the next generation. The process flow of the genetic algorithm may be seen in Figure A.10. The justification for the use of stopping criteria is to ensure that the genetic algorithm produces a solution within a short enough time as the responsiveness of the system is critical. Several stopping criteria are enforced:

- the number of generations created by the chromosome is limited to no more than one hundred times the number of unpicked orders in the system;

- the run time cannot exceed 0.3 seconds;

- the algorithm terminates if over the past 50 generations the average objective function change is less than $1 \times 10^{-6}$ units of distance.

These values were arrived at through testing to find ones which gave a good performance. Initial simulations showed that the genetic algorithm suffered stability issues if the picker had not yet picked any orders, as the picker would get re-routed multiple times without having picked a single item. The minimum number of items to pick from a pick list before updating was set at $20 \%$ of the initial number to resolve this issue. At high values of $N$ the genetic algorithm also had some stability issues. This was rectified by modifying the algorithm so that it could not be intervened at $N=20$. Furthermore, if the next pick list exceeds the 


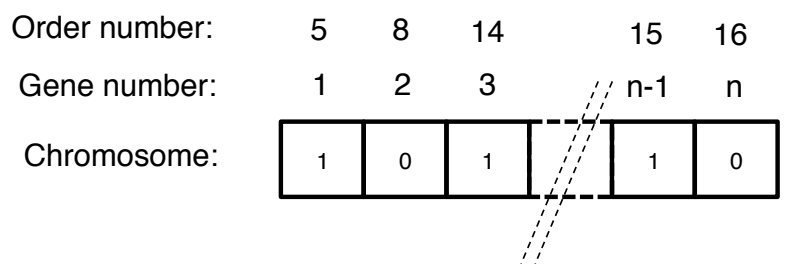

Figure A.9: Example of chromosomes assigned to orders

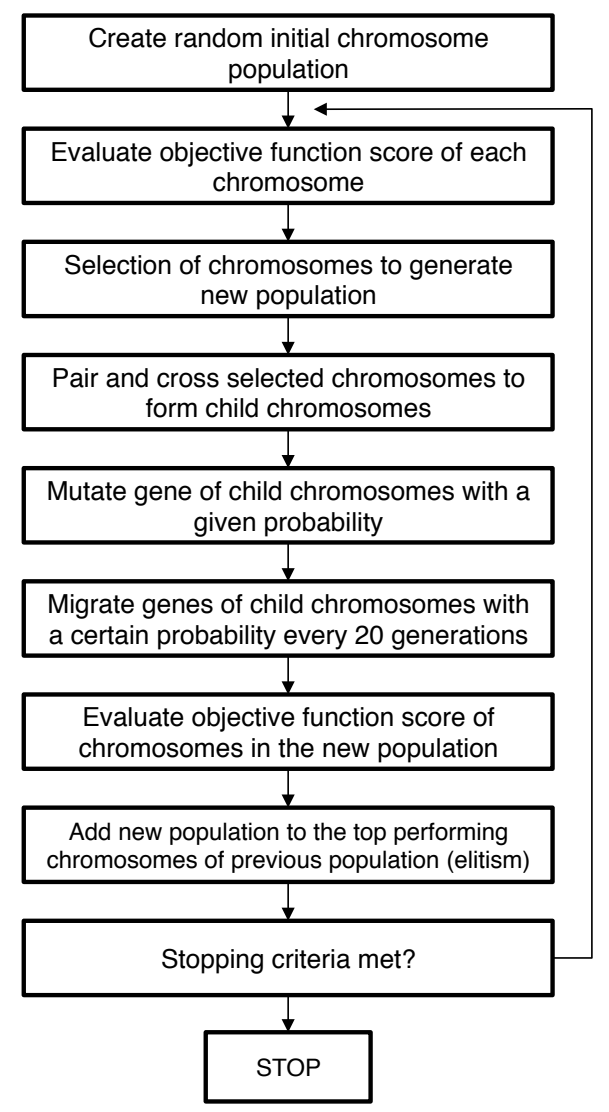

Figure A.10: Process flow of the genetic algorithm

cart capacity, the 20 orders with the oldest arrival rate are used as the starting point for the next pick list to minimise the AOCT. For the interested reader, a more detailed description of this policy can be found in (Robertson, 2015). 